\title{
The Dewey Monitor: Pulse Oximetry can Warn of Hypoxia in an Immersed Rebreather Diver in Multiple Scenarios
}

\author{
Rachel M. Lance $10,{ }^{1,2,4}$ Michael J. Natoli, ${ }^{1}$ Fabio Di Pumpo, ${ }^{3}$ \\ Timothy P. Beck, ${ }^{4}$ Alan Gatrell, ${ }^{1,2}$ Gregory J. Brown, ${ }^{1}$ \\ Derek Schocken, ${ }^{1,4}$ and Richard E. Moon ${ }^{1,2,5}$

\begin{abstract}
${ }^{1}$ Center for Hyperbaric Medicine \& Environmental Physiology, Duke University Medical Center, Durham, NC, USA; ${ }^{2}$ Department of Anesthesiology, Duke University Medical Center, Durham, NC, USA; ${ }^{3}$ Medical Service of Divers and Raiders Group Command "Teseo Tesei” COMSUBIN, Italian Navy, La Spezia, Italy; ${ }^{4}$ Duke University School of Medicine, Durham,
\end{abstract} \\ NC, USA; and ${ }^{5}$ Department of Medicine, Duke University Medical Center, Durham, NC, USA
}

(Received 13 June 2021; accepted 3 January 2022; published online 15 January 2022)

Associate Editor Sean S. Kohles oversaw the review of this article.

\begin{abstract}
Divers who wish to prolong their time underwater while carrying less equipment often use devices called rebreathers, which recycle the gas expired after each breath instead of discarding it as bubbles. However, rebreathers' need to replace oxygen used by breathing creates a failure mechanism that can and frequently does lead to hypoxia, loss of consciousness, and death. The purpose of this study was to determine whether a pulse oximeter could provide a useful amount of warning time to a diver with a rebreather after failure of the oxygen addition mechanism. Twenty-eight volunteer human subjects breathed on a mixed-gas rebreather in which the oxygen addition system had been disabled. The subjects were immersed in water in four separate environmental scenarios, including cold and warm water, and monitored using pulse oximeters placed at multiple locations. Pulse oximeters placed on the forehead and clipped on the nasal ala provided a mean of $32 \mathrm{~s}( \pm 10 \mathrm{~s}$ SD) of warning time to divers with falling oxygen levels, prior to risk of loss of consciousness. These devices, if configured for underwater use, could provide a practical and inexpensive alarm system to warn of impending loss of consciousness in a manner that is redundant to the rebreather.
\end{abstract}

Keywords-Oximeter, Oxygen, Failure, Equipment, Anoxia, Consciousness.

Address correspondence to Rachel M. Lance, Center for Hyperbaric Medicine \& Environmental Physiology, Duke University Medical Center, Durham, NC, USA. Electronic mail: Rachel. lance@duke.edu

\section{INTRODUCTION}

Rebreathers are devices that recycle a diver's exhaled breathing gas rather than venting it, unlike opencircuit SCUBA (Self-Contained Underwater Breathing Apparatus). Rebreathers remove expired carbon dioxide via a passive chemical reaction with a "scrubber" material, and in fully closed rebreather models, more oxygen is injected as it is used by the diver. Pure oxygen rebreathers use a mechanical injection system, but most mixed-gas closed circuit rebreathers (CCRs) use an electronics package comprised of oxygen sensors, a computer, and a solenoid. This package measures and injects oxygen to maintain a preset desired oxygen partial pressure $\left(\mathrm{pO}_{2}\right)$. The oxygen sensing mechanism is therefore simultaneously responsible for both $\mathrm{pO}_{2}$ adjustment and safety monitoring, thus failure can result in either hypoxia (possibly causing loss of consciousness) or hyperoxia (possibly causing seizure due to oxygen toxicity).

Rebreather diving is the activity with the second highest fatality rate in the world per hour of performance, second only to BASE jumping. ${ }^{2}$ Hypoxia is the leading cause of fatality for rebreather divers at $38.9 \%$ of deaths, while in contrast hyperoxia is responsible for only $9.6 \% .^{5}$ While hypoxia has signs and symptoms that can be recognizable, studies by the U.S. Air Force have concluded that personnel are likely to notice the symptoms before loss of consciousness only if those personnel have previously undergone hypoxia training in a controlled environment, as $94 \%$ of untrained pi- 
lots experience loss of consciousness despite education about hypoxia's symptoms. ${ }^{4}$ Hypoxia usually occurs because of a failure within the oxygen measurement and injection system, which can include oxygen-related diver failures, such as forgetting to open the oxygen cylinders that functionally disable the injection systems. All known models of rebreather have at least one failure mode that will cause simultaneous loss of the ability to measure oxygen, automatically inject oxygen, and warn the diver of falling oxygen levels. For example, failure of the oxygen injection solenoid, or in the case of manual rebreathers, failure of the actuator button required to inject more oxygen manually.

Pulse oximetry has been used extensively to monitor blood oxygen saturation $\left(\mathrm{SpO}_{2}\right)$ levels in freedivers as well as personnel in breath-hold scenarios. ${ }^{9,10}$ A previously reported pilot study investigated the utility of a forehead-worn pulse oximeter to detect falling oxygen levels in the rebreather's breathing loop by measuring the blood oxygen saturation $\left(\mathrm{SpO}_{2}\right)$ of the diver rather than the partial pressure of oxygen $\left(\mathrm{piO}_{2}\right)$ inspired from the breathing loop. ${ }^{6}$ The pilot study indicated that a forehead-worn oximeter may provide a minimum of $49 \mathrm{~s}( \pm 17 \mathrm{~s} \mathrm{SD})$ of warning time to the diver independently of the rebreather's electronics package. However, this pilot study was performed with test subjects in the air rather than immersed in water, and thermal changes in water have been shown to cause delays of up to four minutes for pulse oximeters placed at certain measuring locations. ${ }^{7}$

The purpose of the study reported herein was to measure the amount of warning time that could be provided to a diver who has experienced a rebreather failure by pulse oximeters that were placed at various locations on the body, while that diver was fully immersed in water. Divers were tested in both warm and cold water. This project was initiated because of the death of Dewey Smith, a rebreather diver whose electronics package was accidentally turned off by the pressure waves created by nearby underwater tools, causing his death from hypoxia.

\section{MATERIALS AND METHODS}

All testing was conducted with the approval of Duke University Medical Center's Institutional Review Board (DUMC IRB, Pro \#00060088). Test subjects were compensated volunteers who had given written informed consent. Each test subject was evaluated prior to enrollment using prescreening that determined their maximum oxygen consumption level $\left(\mathrm{VO}_{2 \max }\right)$ and fitness to dive. Test subjects qualified for the study if there were determined to be representative of the military diving population, defined as able to achieve a $\mathrm{VO}_{2 \max }$ of $35 \mathrm{~mL} / \mathrm{kg} /$ minute for males or 30 $\mathrm{mL} / \mathrm{kg} / \mathrm{minute}$ for females, in the age range 18-40, and medically fit to dive. Subjects were also required to be neither smokers nor pregnant, with negative pregnancy determined by blood test.

The rebreather used for this study was a Megalodon (InnerSpace Systems Corporation, Chehalis, WA) with a radial-flow carbon dioxide scrubber capable of holding $3.6 \mathrm{~kg}(8 \mathrm{lb}$ model $)$ of carbon dioxide absorbent material. This design configuration is in use by the U.S. Navy as the UBA Mk 28 (Underwater Breathing Apparatus Mark 28). The rebreather was modified to include two three-way valves (Hans Rudolph model $2100 \mathrm{~A}$, Shawnee, KS) in the breathing loop, one on each side of the mouthpiece (Fig. 1). By turning these valves, the dive tender could switch the source of the diver's breathing gas and the direction of their exhaled gas. As the valves has an internal flow bore $(22.2 \mathrm{~mm}$, 0.9 inches) that was almost identical to the minimum internal diameter within the rebreather breathing loop (1 inch), they were not expected to restrict gas flow over the inherent work of breathing of the rebreather. The maximum expected differential pressure across the valve was $0.07 \mathrm{cmH}_{2} \mathrm{O}$ per the manufacturer. ${ }^{3}$

In the first valve configuration, referred to as the "open" configuration, the valves provided the subject with air from a corrugated breathing hose, and the subjects exhaled through the rebreather mouthpiece into a second hose that could be either vented to the room or emptied into a 200-L Douglas bag. In the second configuration, referred to as the "closed" configuration, the valves kept all of the breathing gas recirculating within the closed breathing loop. In the closed configuration, the valves provided the divers with gas from the rebreather breathing loop, and also caused them to exhale back into the rebreather breathing loop, creating a "closed loop" similar to how the rebreather loop would operate in a real-world diving scenario. The valves were determined to add 30 $\mathrm{mL}$ each of volume to the Megalodon breathing loop, which had a measured internal gas volume of $13.5 \mathrm{~L}$ after subtraction of the calculated volume filled by the solid granules of the scrubber material (calculated to be $4030 \mathrm{~mL}^{8}$ ).

Prior to each study, the rebreather's carbon dioxide scrubber was filled with $3.6 \mathrm{~kg}( \pm 0.09 \mathrm{~kg})$ of Sofnolime 797 grade 8-12 mesh (Molecular Products, Boulder, CO USA). Both of the electronics packages on the rebreather were switched to "off" so that there would be no alarms or activation of the oxygen addition solenoid. Compressed bottles of oxygen and diluent, which are normally part of the rebreather configuration, were omitted from the setup as the rebreather was switched off and therefore would not need to inject either gas. A sampling port was inserted 


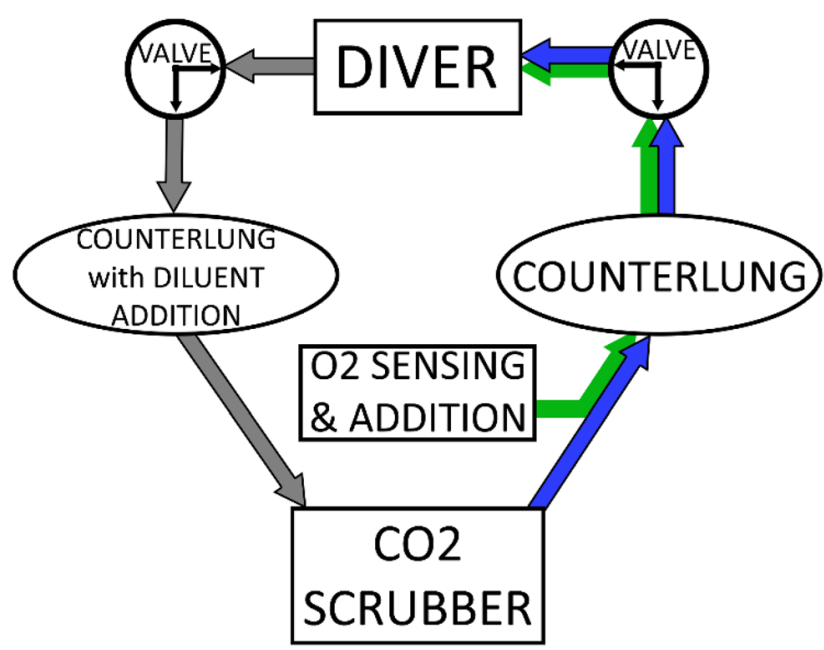

FIGURE 1. Diagram of the Innerspace Megalodon breathing loop, with added valves. All known mixed-gas rebreathers share the same functional components of scrubber, counterlung(s), and gas addition, although some vary in their exact configuration and methods of adding oxygen and diluent gases. The three-way valves are not a typical part of rebreather design, were inserted for this study, and are shown in the "closed loop" configuration in this diagram. If both valves were rotated $90^{\circ}$ (clockwise on inhale side, counter-clockwise on exhale side), the diver's intake and exhaust would connect directly to room air instead of to the counterlungs.

into the breathing loop immediately upstream of the mouthpiece, which provided a continuous sample of the inspired breathing gas to a pre-calibrated mass spectrometer (Perkin Elmer model MGA 1100 [refurbished by MA Tech Services], Waltham, MA). The mass spectrometer was calibrated both before and after testing using multiple calibration gas compositions and examining the response data for appropriate step increases and plateaus at equilibrium. The volume lost to sampling was determined to be negligible in comparison to the total loop volume. The output from the mass spectrometer was monitored and recorded using LabChart software (version Pro 7, AD Instruments, Colorado Springs, CO, USA).

The sites at which pulse oximeters were located on the test subjects were the forehead, sternum, scapula, mastoid process, and clipped on the nasal ala. The sites used for each subject are listed in detail in the Results section, and the sites varied for each subject because oximeters were added to new locations as new devices became available, or removed once configurations or sites were shown to be non-viable. The oximeters used on the sternum, scapula, and mastoid process were reflectance models (Nonin model 7500, Nonin Medical, Inc., Plymouth, MN USA) connected to a receiver unit (Nonin model 3150, Nonin Medical, Inc., Plymouth, MN USA) that was both recording the data and transmitting it via Bluetooth to a custom Matlab program (version R2016b, MathWorks, Natick, MA USA). The Matlab program was used to monitor the output in real-time. The first four test subjects were instrumented on the forehead using this same sensor configuration, while later subjects were instrumented on the forehead with a Nellcor reflectance sensor (Nellcor SpO2 Forehead Sensor with OxiMax Technology, Medtronic, Minneapolis, MN USA) connected to a Nellcor monitor (Nellcor Portable SpO2 Patient Monitoring System, PM10N, Medtronic, Minneapolis, MN USA). The Nellcor reflectance sensor was incorporated into the skirt of a SCUBA half mask. This device was the same one described and used in Ref. 1. Subjects were monitored on the nasal ala with a clipstyle sensor that clipped onto the site (Nasal Alar SpO2 Sensor, Tri-anim, Dublin, OH USA) and connected with the same model monitor (Nellcor PM10N) as the forehead sensor. All $\mathrm{SpO} 2$ monitors had a sampling frequency of $1 \mathrm{~Hz}$. Nonin reflectance sensors were waterproofed with a combination of potting with clear materials and application with Tegaderm adhesive stickers. Efforts were made to ensure that no waterproofing materials covered the functional surface of the sensors, and sensors were validated for accuracy after waterproofing by comparison with a non-modified identical model during voluntary apnea. The Trianim nasal alar sensor had an inherently waterproof sensor head that needed no modification for use in water, but its cable junctions were waterproofed with application of epoxy and coating with Plasti-Dip (Plasti Dip International, Blaine, MN).

Test subjects were immersed face-down in a water tank that was positioned inside a hyperbaric chamber. A dive "tender" was present inside the water tank at all times to monitor the subjects for safety and to assist them with the equipment. Before the experiment, each 
subject was fitted with an arterial blood gas (ABG) line, inserted into the radial artery of the arm selected by the subject. A second qualified researcher was positioned inside the hyperbaric chamber but outside the water tank; this researcher drew blood gas samples before the start of each hypoxic period, and at the termination point of each hypoxic period. Subjects were also instrumented with a 3-lead ECG. Research personnel continuously monitored the ECG during the experiments using the same LabChart software as was used to monitor the mass spectrometer output.

Each test subject underwent four experimental sequences, which were designed to elucidate the impacts of water temperature, depth, and exercise level on the length of warning time provided by the sensors at each location. The testing protocol was the same for each of the four experimental sequences, with the environmental variables changed for each sequence. Sequence 1 took place in cold water $\left(20-21^{\circ} \mathrm{C}\right)$ with a high rate of work (targeted oxygen consumption level $2 \mathrm{~L} / \mathrm{min}$ ) at surface pressure ( 0 feet of seawater, fsw). Sequence 2 took place in cold water with a moderate rate of work (targeted oxygen consumption level $1 \mathrm{~L} / \mathrm{min}$ ) at surface pressure. Sequence 3 took place in warm water $(28$ $30{ }^{\circ} \mathrm{C}$ ) with a high rate of work at surface pressure. For Sequence 4, the hyperbaric chamber was pressurized to the equivalent depth of $77 \mathrm{fsw}$, with the subject in warm water exercising at a high work rate. The rate of work was calculated using the results of each subject's $\mathrm{VO}_{2 \max }$ testing, which allowed the selection of the external load that should be placed on a bicycle ergometer to achieve the desired level of oxygen consumption.

Sequences 1 and 2 were performed as closely together as possible to minimize thermal exposure for the subjects. They were typically separated by about ten minutes, the length of time required to purge and reset the equipment. Between Sequence 2 and 3, the water tank was drained and refilled with warm water, which took roughly $2 \mathrm{~h}$. The subjects were allowed to warm during this time period. Sequence 4 was then conducted as soon as possible after Sequence 3, approximately ten to fifteen minutes later. It is possible that there was an order effect from this timing; however, the sequence was selected to minimize the risk of decompression to the subjects, as risk of decompression sickness is altered based on the timing of exposure to different water temperatures.

For each test sequence, the subject donned the rebreather, immersed face-down in the water tank, and positioned their feet in the pedals of an underwater bicycle ergometer. For the first four test subjects, the subjects used a full face mask (Interspiro Divator, Interspiro, Stockholm, Sweden) connected to the Megalodon mouthpiece to mimic a US Navy re- breather diving configuration. For reasons described in more detail in sections below, this configuration proved experimentally problematic and was abandoned. The remaining twenty-four subjects wore a conventional SCUBA half-mask and held the Megalodon "T-bit" mouthpiece in their mouths. The in-tank tender positioned the breathing loop valves in the "open" configuration so that the subject was breathing room air through the mouthpiece, and exhaling to room air. The subject pedaled on the ergometer at 60 RPM for five minutes to achieve a steady state of oxygen consumption. The subject then continued to pedal on the ergometer for an additional minute, while still breathing room air, as their expired gas was collected in a Douglas bag to allow later validation of their oxygen consumption rate. During the one-minute duration of the gas collection period, the out-of-tank tender drew a baseline arterial blood gas sample to validate the starting level of blood oxygen saturation. Following gas collection, the in-tank tender switched the valves to the "closed" configuration so that the subject was rebreathing from the closed breathing loop. The time period when the subjects were breathing in the "closed" configuration is referred as the hypoxic period, as no oxygen addition into the breathing loop was possible during this period. During the test sequences, beginning with the start of the exercise period, the subjects were asked to respond to a light in the water tank that blinked at a randomized sequence. The subjects responded to the light by depressing a button, and both the light activation and subsequent button activation were monitored for subject responsiveness by personnel outside the chamber using LabChart.

The subjects continued to breathe from the closed breathing loop during the hypoxic period while pedaling at the same rate until they reached the first of any of four termination criteria. The hypoxic period was terminated when either: (1) $\mathrm{SpO}_{2}$ levels on any properly functioning pulse oximeter reached $80 \%$, (2) inspired $\mathrm{pO}_{2}\left(\mathrm{piO}_{2}\right)$ measured by the mass spectrometer reached 0.12 atmospheres, (3) the subject failed once to press the button in response to the light during the hypoxic period, or (4) the subject voluntarily selfterminated. "Properly functioning" pulse oximeters were defined as oximeters that had provided $\mathrm{SpO}_{2}$ levels within reasonable ranges during the normoxic exercise period, without error codes. An arterial blood gas sample was drawn as closely as possible to the time of the termination. The in-tank tender ended the hypoxic period by rotating the valves to the "open" configuration so that the subject was breathing room air, and the subjects were monitored until their $\mathrm{SpO}_{2}$ levels returned to normal. Following the conclusion of each test sequence, the collected expired gas was measured to determine exhaled gas volume and frac- 
tions of oxygen and carbon dioxide, which were used to calculate the oxygen consumption rate of the subject.

Following each experiment, raw data from the pulse oximeters were downloaded using the proprietary software provided by each of the respective manufacturing companies. The data were aligned in time sensors using the time stamps with custom software written in Matlab (version R2020a, The MathWorks, Inc., Natick, MA, USA). The available warning time was defined as the number of seconds between the time when the measured $\mathrm{SpO}_{2}$ dropped below $95 \%$ and stayed below that level, and the time when the test was terminated because of one of the four criteria listed above. The value of $95 \%$ was selected for consistency with Ref. 6 , which determined $95 \%$ to be a desirable level to avoid false positives for a $\mathrm{SpO}$-based hypoxia alarm.

Variable selection was performed by multivariate ANOVA (JMP Pro 15.1.0, SAS Institute Inc., Cary, NC USA) with warning time as the output variable, and water temperature, $\mathrm{VO}_{2}$, depth, and sensor locations as the input variables. Although $\mathrm{VO}_{2}$ was determined not to be a statistically significant predictor of warning time by the ANOVA, a linear regression line was nonetheless fit with $\mathrm{VO}_{2}$ as the independent variable so that the level of correlation could be examined through the Pearson correlation coefficient (model fit in Microsoft Excel, Version 2105, Microsoft Corporation, Redmond, WA, USA).

\section{RESULTS}

Twenty-eight human volunteer subjects were tested in the experimental protocol. The final two planned subjects were eliminated from the test series because of limitations from the COVID-19 pandemic.

The first four subjects were tested using a slightly different protocol than the remaining 24 subjects; these negative results are included briefly here for the purpose of communicating the issue to future investigators who wish to use this configuration. The first four subjects were testing using a full face mask attached to the rebreather breathing loop, rather than a conventional SCUBA half mask and T-bit mouthpiece. However, the goodness of the fit of the full face mask varied with the differing shapes of the faces of test subjects, and each subject's goodness of fit proved to dictate the utility of the forehead pulse oximeter for that subject. Two of the subjects showed adequate readings from the forehead pulse oximeter while wearing the full face mask, but the other two subjects showed fluctuations in $\mathrm{SpO}_{2}$ measurement that correlated with immersing or removing their faces from the water, despite continuously breathing normoxic room air. On immersion, $\mathrm{SpO}_{2}$ would drop to $75-85 \%$, then rebound when the subject raised their head back out of the water. Subjects reported no discomfort from the mask skirts.

The impact of this effect on the data in addition to the experimental difficulties of having untrained divers use both a full face mask and a rebreather in cold water led to the elimination of the full face mask from the experimental setup.

The remaining 24 subjects were tested using a traditional SCUBA half mask and rebreather T-bit.

Locations on the scapula, sternum, and mastoid process proved to be of limited utility because of the susceptibility of these sensor locations to changes caused by immersion, water temperature, exercise status, and motion artifacts from the equipment. Therefore, data from these locations were not included in the quantitative analyses of warning time. Representative traces from each of these sensor locations are shown as Fig. 2

The sensor locations that proved to be most useful were the forehead and nasal ala, which together yielded a total of 91 measurements of the available warning time four sets of experimental conditions. The measured warning times from the forehead and nasal ala ranged from a minimum of 17 seconds to a maximum of 58 seconds. Representative traces from the forehead and nasal sensor are shown for all four test sequences in Fig. 3. All data for this project, including all data traces from all sensor locations for each experimental sequence, have been uploaded and made available online via the Duke Research Data Repository (http s://research.repository.duke.edu/).

Consistently, the sensors provided the cleanest data traces when at increased pressures, likely because of the increased partial pressure of oxygen.

The results of the ANOVA for the warning times from the forehead and nasal ala indicated that none of the suspected predictive variables (sensor location, $\mathrm{VO}_{2}$, depth, and water temperature) were statistically predictive of the amount of warning time available ( $\mathrm{p}=0.56,0.15,0.67$, and 0.91 respectively). The warning times for all tests are shown as a function of oxygen consumption $\left(\mathrm{VO}_{2}\right)$ in Fig. 4. The linear regression line had a Pearson correlation coefficient of 0.15 , supporting the lack of relationship for this experiment.

No statistical relationship was found that suggested that any of the variables were dictating the available warning time. The mean value of the warning times for the 91 usable trials was $32 \mathrm{~s}$ ( $\pm 10 \mathrm{~s}$ standard deviation), and the median warning time was $30 \mathrm{~s}$. The warning times are shown along with other key experimental data in Tables 1 and 2. Ideally, 24 subjects 

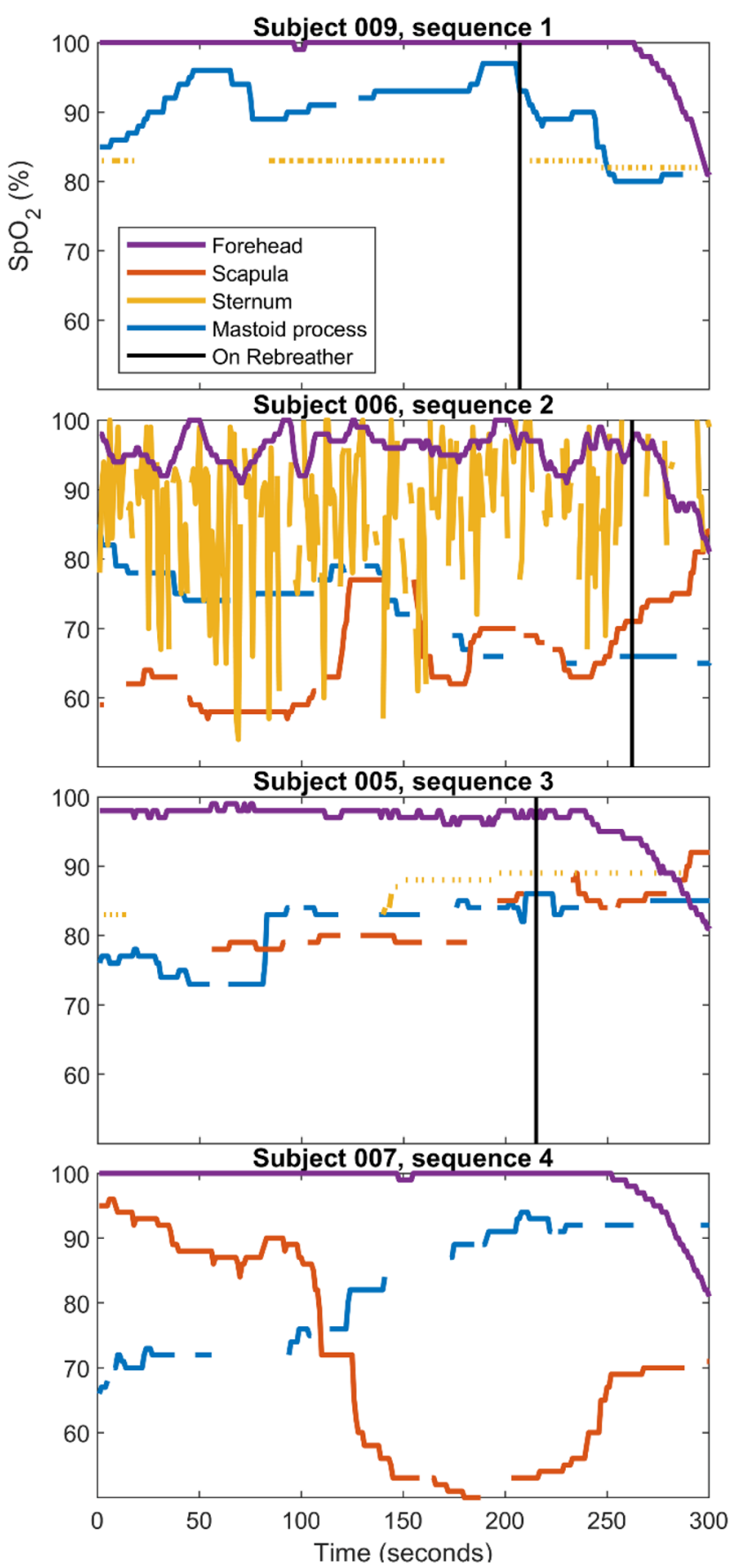

FIGURE 2. Representative traces from sensors located on the sternum, scapula, and mastoid process. One curve from each test sequence is shown. Data were selected from the subjects with the most intact data from the sternum, scapula, and mastoid locations for each of those locations. The vertical black lines indicate the time point that the subjects were switched from room air to the closed rebreather loop. While subjects were put on the rebreather at the same point within each experimental sequence, the hypoxic period lasted variable lengths of time before the experiment reached a termination point. Since the upper limits of the $x$ axes denote the termination points for each experiment, the variable-length hypoxic periods mean the black lines will appear at variable positions along the time scale.

undergoing 4 sequences with 2 sensors would have resulted in 192 measurements total. However, nasal alar sensors were not available for the first 12 subjects, and thus were not used. In addition, the experimental difficulties of the test setup meant that test failures were unfortunately common. The most common causes of failure were thermal issues for test subjects in the cold water, leading to failure to complete the test se- 

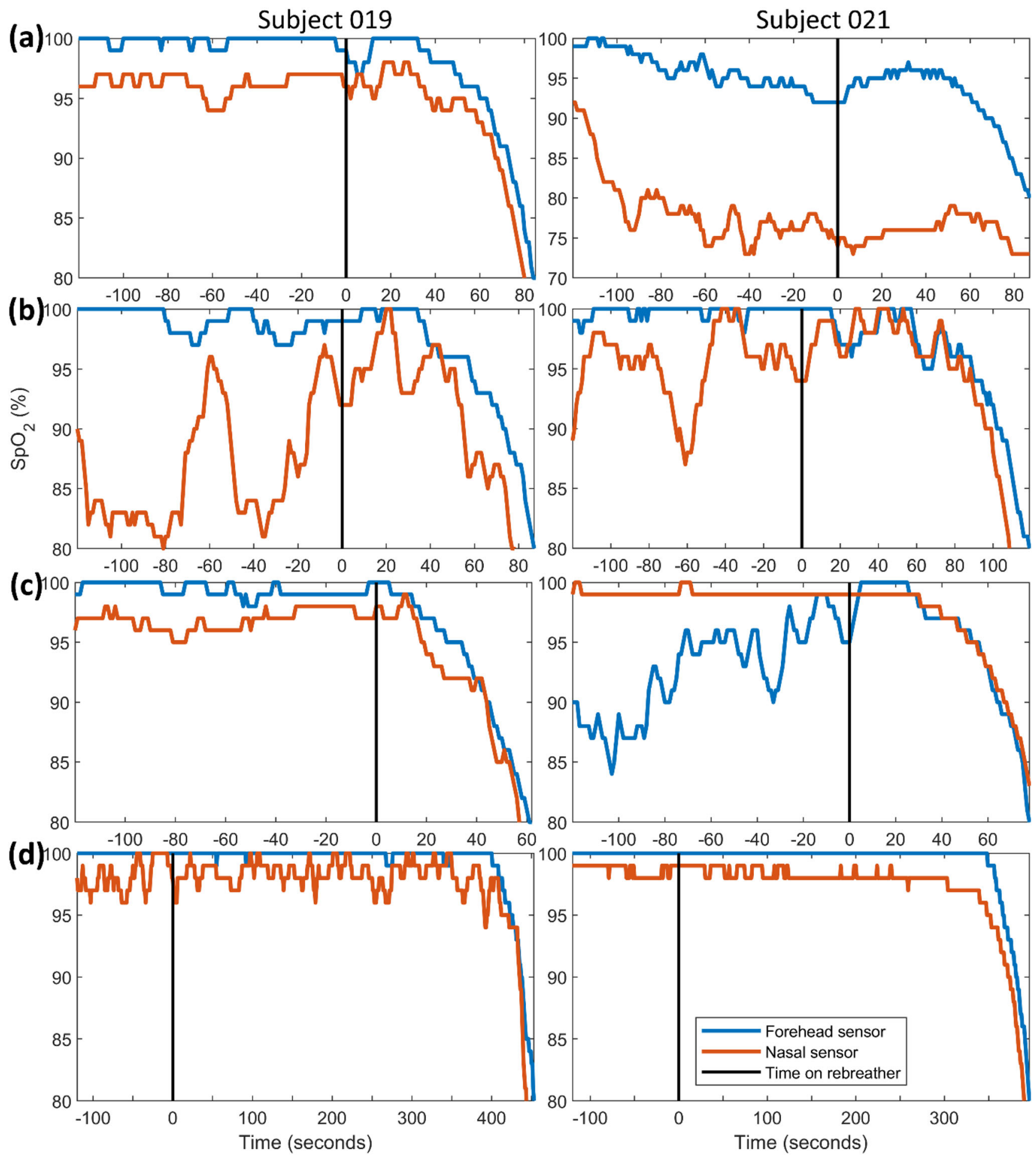

FIGURE 3. Representative $\mathrm{SpO}_{2}$ data traces from the forehead and nasal sensor. (a) Test sequence 1: cold water, moderate work rate (b) Test sequence 2: cold water, light work rate (c) Test sequence 3: warm water, moderate work rate (d) Test sequence 4: warm water, moderate work rate, chamber pressurized to $77 \mathrm{fsw}$. Note that several of the traces from sequences at surface pressure (a-c) show artifacts from sensors that have been dislodged, affected by water intrusion, or are experiencing normal artifacts from motion. The data set in this figure was selected to provide a broad range of curves, from those where the sensors were successful (e.g., 019d and 021d) and also those where the sensors yielded problematic results that indicated a need for improved sensor engineering (e.g., 021a and 019b nasal traces). Both the quantity and the magnitude of artifacts were greatly diminished in the data traces at depth for all subjects, likely because of the increase partial pressure of oxygen. Please see note in Fig. 2 caption regarding the variable location of the vertical black lines that indicate the time the subject was switched to the rebreather. 


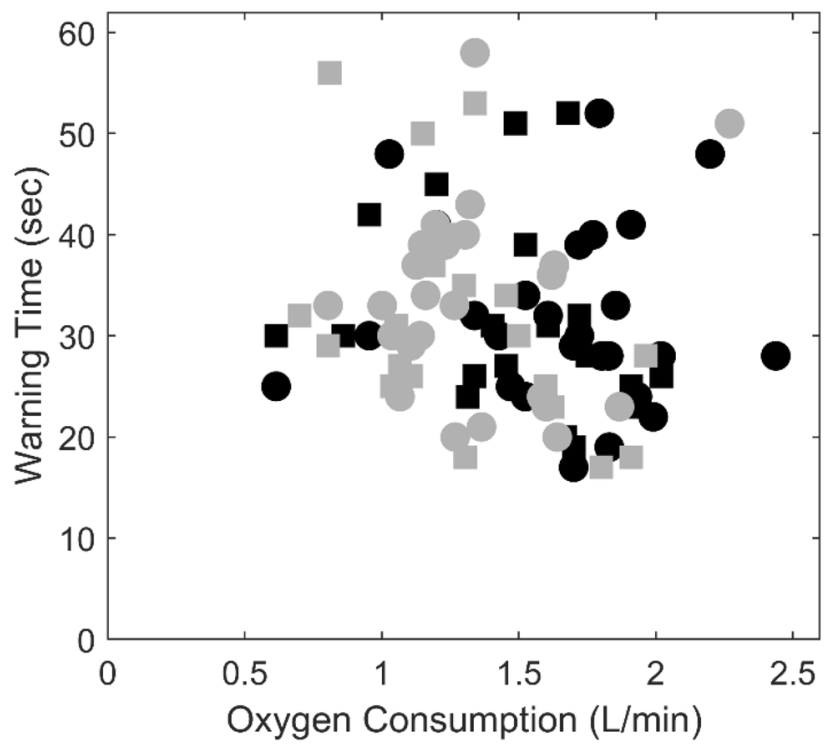

FIGURE 4. Warning times plotted compared to rate of oxygen consumption. Circles indicate a warning time that was measured by a sensor placed on the forehead, squares a sensor on the nasal ala. Black markers indicate the test was conducted in warm water, grey indicates cold water.

quences, and engineering equipment failures that could not be addressed while leaving the subject in the water tank. All data points for which the subjects completed the hypoxic period with at least one working sensor are reported in the Tables, without exclusion.

\section{DISCUSSION}

A mean value of $32 \mathrm{~s}$ is sufficient time for rebreather divers to take any one of several possible corrective actions prior to loss of consciousness, such as manually adding diluent gas or oxygen to their breathing loop via depressing an onboard button. All known models of rebreather have a mechanism to add oxygen with a single button press. Such a monitor may also provide sufficient time to allow more complex corrective measures such as communicating the need for assistance from a dive buddy, or switching their gas source to a tank of compressed gas; however, test subjects were not evaluated on more complex actions as part of this study. These results are consistent with our pilot study, which also showed that a pulse oximeter can provide a useful warning device for rebreather divers with failed equipment in a diving accident.

The shorter-duration time points (e.g., $17 \mathrm{~s}$ for test subject 27 test sequence 1) seemed to correlate to a higher level of mask-clearing activity by the subjects. During all experiments, subjects cleared accumulated water from their masks by exhaling through their noses into the mask, which meant that some of the gas and therefore oxygen from the breathing loop was lost and exhaled into the water tank as bubbles out of the mask with each clearing action. Some subjects cleared their masks noticeably more often, which seems as if it may have been correlated with shorter available warning times. For example, it was noted during the experiment that subjects 24 and 27 seemed to be clearing their masks more often than most other subjects. During later analysis, these subjects were calculated to have some of the shortest available warning times $(25,18$, 19 , and $25 \mathrm{~s}$ for subject $24 ; 17,18$, and $20 \mathrm{~s}$ for subject 27 with a failure to complete the final test sequence). However, while it makes logical sense that loss of breathing gas through mask clearing would shorten the available warning time, these experiments did not include a quantification of lost gas volume, so it was not possible to separate out such data points in a robust and non-subjective fashion. The lack of statistical relationship of rate of oxygen consumption with warning time was a result that was unexpected, but could possibly be explained by mask clearing having a larger influence on the total rate of loss of oxygen from the loop.

The major limitations of this work were the dual experimental challenges of having naïve test subjects use complex rebreathing equipment underwater while in cold water, and ensuring proper functionality of non-factory-waterproofed sensors underwater during the experiments. These challenges led to gaps in the dataset, which are more obvious in the reduced number of successful data points in the cold-water data in Table 1 compared to the warm-water data in Table 2 . All of the sensors used required some version of a wired connection to an external monitoring unit, which inherently created a vulnerability in the experimental test setup that often led to equipment failure from water intrusion.

A possible limitation for application of this work to the broader civilian population is our selection of divers designed to be representative of the military diving population. Should this work be used to inform the creation of a safety-related monitor, it should be expanded to assess whether results vary with a broader spectrum of physiologies.

The first four data points used a forehead reflectance pulse oximeter inside a full face mask. This design proved problematic because only one model of Interspiro full face mask is available. While all subjects were tested during screening for their ability to wear the face mask and dive with it comfortably in the experimental water tank, these screening tests were conducted with the mask attached to an open-circuit gas source. The use of an open-circuit gas source provided the subjects with an effectively unlimited source of pressurized gas that was piped into the mask 
TABLE 1. Experimental data and warning times for the cold water trials, test sequences \#1 and 2.

\begin{tabular}{|c|c|c|c|c|c|c|c|c|}
\hline \multirow[b]{2}{*}{ Subject } & \multicolumn{4}{|c|}{ Sequence 1: moderate work rate } & \multicolumn{4}{|c|}{ Sequence 2: light work rate } \\
\hline & Warning time (s) & sO2 at baseline & $\mathrm{sO} 2$ at end & VO2 (L/min) & Warning time $(\mathrm{s})$ & sO2 at baseline & $\mathrm{sO} 2$ at end & VO2 (L/min) \\
\hline 5 & & & & & 30 & & & 1.1 \\
\hline 6 & & & & & 33 & 98.9 & & 1.0 \\
\hline 7 & 51 & 98.7 & 79.4 & 2.3 & & & & \\
\hline 8 & 23 & 99.4 & 69.9 & 1.6 & 34 & 99.1 & 88.9 & 1.2 \\
\hline 9 & 23 & 99.4 & 70.2 & 1.9 & 21 & 99.2 & 73.8 & 1.4 \\
\hline 10 & 36 & 98.8 & 73.0 & 1.6 & 37 & 98.9 & 85.5 & 1.1 \\
\hline 12 & 34 & 99.1 & 60.0 & 1.5 & 20 & 98.6 & 77.5 & 1.3 \\
\hline 13 & 33 & 97.2 & 79.1 & 1.3 & 33 & 96.7 & 78.9 & 0.8 \\
\hline 13 & 40 & 97.2 & 79.1 & 1.3 & 29 & 96.7 & 78.9 & 0.8 \\
\hline 14 & 40 & 98.2 & 76.8 & 1.3 & 39 & 98.1 & 76.7 & 1.1 \\
\hline 14 & & & & & 50 & 98.1 & 76.6 & 1.1 \\
\hline 15 & 41 & 97.0 & 79.8 & 1.2 & & & & \\
\hline 16 & & & & & 39 & & & 1.2 \\
\hline 17 & 20 & 95.6 & 68.7 & 1.6 & 24 & 72.4 & 96.9 & 1.6 \\
\hline 19 & 24 & 97.3 & 76.2 & 1.1 & 29 & 97.5 & 74.7 & 1.1 \\
\hline 19 & 27 & 97.3 & 76.2 & 1.1 & 26 & 97.5 & 74.7 & 1.1 \\
\hline 20 & 37 & 98.9 & 75.4 & 1.6 & & & & \\
\hline 21 & 39 & 97.2 & 71.4 & & 30 & 97.8 & 72.6 & 1.0 \\
\hline 21 & & & & & 25 & 97.8 & 72.6 & 1.0 \\
\hline 22 & 28 & 98.9 & 70.1 & 2.0 & 53 & 98.4 & 73.9 & 1.3 \\
\hline 22 & & & & & 58 & 98.4 & 73.9 & 1.3 \\
\hline 23 & 30 & & & 1.5 & 37 & & & 1.2 \\
\hline 24 & 25 & & & 1.6 & 18 & & & 1.3 \\
\hline 25 & 35 & 98.7 & 72.6 & 1.3 & & & & \\
\hline 26 & 23 & & & 1.6 & 31 & & & 1.1 \\
\hline 27 & 17 & 97.9 & 58.1 & 1.8 & 18 & 97.8 & 70.4 & 1.9 \\
\hline 28 & 32 & 99.2 & 76.7 & 0.7 & 56 & 98.8 & 82.0 & 0.8 \\
\hline
\end{tabular}

Sequence 1 had a targeted oxygen consumption rate of $2 \mathrm{~L} / \mathrm{min}$, sequence 2 had a targeted rate of $1 \mathrm{~L} / \mathrm{min}$. Both sequences were performed at surface pressures. Warning time is defined as the number of seconds between the time point when the sensor drops and stays below $95 \%$ and the time point when the test was terminated, which was most commonly at $\mathrm{SpO}_{2}=80 \%$. Reported $\mathrm{sO}_{2}$ values are the results of the arterial blood-gas analysis at the timepoints before transition to the rebreather, and at the test termination timepoint. Italic values are experiments with the nasal alar sensor. Roman values cells are experiments with the forehead sensor. None of the first four subjects managed to complete the cold-water trials before the onset of thermal concerns. Details regarding the experimental issues that led to each data point failure are included with the published raw data, see link to Duke Repository in text above. No data points were omitted so long as they reached the end of the test while at least one sensor was functional.

via a conventional SCUBA regulator, then exhaled into the water as bubbles. Testing the subjects on open circuit meant that, if there was a minor leak of water into the mask caused by poor mask fit, any slowly intruding water would be expelled from the mask along with expired gas during normal breathing. However, when the full face mask was attached to the closed circuit rebreather, minor water leaks instead entered the rebreather breathing loop, or the exhalation tube when the loop was in the open configuration. To use the rebreather successfully in conjunction with the full face mask, two of the four subjects needed to tighten the straps for a more secure fit. While all four subjects were able to dive on the rebreather successfully while using the full face mask in a way that limited water intrusion, the two of the four subjects with poorer mask fit showed substantial fluctuation in the $\mathrm{SpO}_{2}$ reading that correlated with the immersion of their faces. Since they were freely breathing normoxic gas during the fluctuations, these fluctuations seem to be caused by the mask skirt limiting blood circulation to the forehead rather than systemic lack of oxygen. Immersing the face while wearing a full face mask places a level of additional pressure on the mask skirt that is noticeable to the diver wearing the mask, which seems to have caused a reduction in blood flow, leading to localized readings of hypoxia for some divers. For any future measurement device that obtains $\mathrm{SpO}_{2}$ measurements from the forehead while using a full face mask, goodness of fit of the full face mask must be a factor. This same phenomenon did not occur when the subjects used a traditional SCUBA half mask, which places less pressure on the skirt of the mask.

Compared with other sites, the sensor locations on the scapula, sternum, and mastoid process proved to be too dependent on the influences of immersion status, water temperature, exercise status, and motion to be pragmatic locations for a warning device for the 
TABLE 2. Experimental data and warning times for test sequences \#3 and 4, warm water trials.

\begin{tabular}{|c|c|c|c|c|c|c|c|c|}
\hline \multirow[b]{2}{*}{ Subject } & \multicolumn{4}{|c|}{ Sequence 3: moderate work, surface pressure } & \multicolumn{4}{|c|}{ Sequence 4: moderate work, 77 fsw } \\
\hline & Warning time (s) & sO2 at baseline & $\mathrm{sO} 2$ at end & VO2 (L/min) & Warning time $(\mathrm{s})$ & sO2 at baseline & sO2 at end & VO2 (L/min) \\
\hline 3 & 29 & & & 1.7 & 17 & & & 1.7 \\
\hline 5 & 43 & 98.6 & 80.6 & & & & & \\
\hline 6 & 25 & 97.6 & 74.7 & 1.5 & & & & \\
\hline 7 & 28 & 98.2 & 69.8 & 2.0 & 28 & 99.0 & 77.2 & 2.4 \\
\hline 8 & 39 & 99.0 & 72.6 & 1.7 & 19 & 99.6 & 65.6 & 1.8 \\
\hline 9 & 41 & 99.2 & 64.3 & 1.9 & 28 & 99.7 & 87.1 & 1.8 \\
\hline 10 & 40 & 98.2 & 72.9 & 1.8 & 33 & 99.8 & & 1.9 \\
\hline 12 & 30 & 99.0 & 82.5 & 1.4 & & & & \\
\hline 13 & 32 & 96.6 & 78.2 & 1.6 & 52 & 98.8 & 85.5 & 1.8 \\
\hline 13 & 31 & 96.6 & 78.2 & 1.6 & & & & \\
\hline 14 & 32 & 98.4 & 72.6 & 1.3 & 28 & 100.0 & 73.3 & 1.8 \\
\hline 14 & 26 & 98.4 & 72.6 & 1.3 & & & & \\
\hline 15 & 48 & & & 1.0 & 48 & 99.4 & 99.1 & 2.2 \\
\hline 16 & 41 & 97.9 & 76.8 & 1.2 & 24 & 98.7 & 88.0 & 1.5 \\
\hline 17 & 24 & 75.9 & 97.8 & 1.9 & 22 & 100.0 & & 2.0 \\
\hline 17 & 23 & 75.9 & 97.8 & 1.9 & & & & \\
\hline 19 & 34 & 97.3 & 74.0 & 1.5 & 30 & 98.1 & 74.7 & 1.7 \\
\hline 19 & 39 & 97.3 & 74.0 & 1.5 & 32 & 98.1 & 74.7 & 1.7 \\
\hline 21 & 25 & 97.6 & 77.8 & 0.6 & 30 & 98.2 & 84.5 & 1.0 \\
\hline 21 & 30 & 97.6 & 77.8 & 0.6 & 42 & 98.2 & 84.5 & 1.0 \\
\hline 22 & 26 & 97.9 & 69.1 & 2.0 & & & & \\
\hline 23 & 52 & & & 1.7 & 28 & & & 1.8 \\
\hline 24 & 19 & & & 1.7 & 25 & & & 1.9 \\
\hline 25 & 24 & 98.6 & 69.0 & 1.3 & 27 & 99.3 & 91.4 & 1.5 \\
\hline 26 & 31 & & & 1.4 & 51 & & & 1.5 \\
\hline 27 & 20 & 97.4 & 65.5 & 1.7 & & & & \\
\hline 28 & 30 & 98.9 & 73.6 & 0.9 & 45 & 98.9 & 99.6 & 1.2 \\
\hline
\end{tabular}

Both sequences had a targeted oxygen consumption rate of $2 \mathrm{~L} / \mathrm{min}$. Sequence 3 was conducted at surface pressure, while sequence 4 was conducted with the chamber pressurized to 77 feet of seawater. Warning time is defined as the number of seconds between the time point when the sensor drops and stays below $95 \%$ and the time point when the test was terminated, most commonly at $\mathrm{SpO}_{2}=80 \%$. Italic values are experiments with the nasal alar sensor. Roman values are experiments with the forehead sensor. See caption for Table 1 for further details.

purposes of these experiments. However, several of the sensors did indicate they could plausibly provide warnings at these locations, especially the scapula, if properly thermally insulated and guarded against motion artifacts. The signal processing required to construct an accurate, robust sensor for those locations was beyond the scope of this project, but the raw data files have been included in the data repository for access and review by other researchers interested in those sensor locations.

Both the forehead and nasal ala proved to be reasonable locations for a sensor, and were not susceptible to issues that may have been caused by water temperature or work rate. As they are located inside the protective volume of the mask, they have the potential to be locations that are relatively resistant to motion artifacts as long as the sensors used are robust to actions such as mask clearing. However, signal processing of the photoplethysmogram will need to be resistant to diver motion and mask clearing or equal- ization activities for any practical device development as the result of this work. Other areas of the body, such as the scapula, are at higher risk for direct impact and thus artifact. In practice, the nasal alar sensor always began to show a drop in $\mathrm{SpO} 2$ before the forehead sensor, which could extend the available warning time. Owing to delayed detection of hypoxemia in cold conditions, use of a finger probe is unlikely to be useful in this setting. ${ }^{7}$ No theoretical reason exists for the rate of decrease in $\mathrm{SpO}_{2}$ to change with increasing atmospheric pressure. However, rebreathers demand an increase in the level of work from the diver in order to use them to breathe an increased depths. ${ }^{11}$ Therefore, one pressurized test sequence was included in this experiment to assess whether that increased work of breathing would cause an alteration in the breathing gas dynamics that dictated the available warning time. No discernible difference could be found using statistical analysis or anecdotal observation; however, the depth experiments did provide one other valuable re- 
sult. Many of the motion and signaling artifacts present on the surface disappeared at depth (see Fig. 3). When under pressure, test subjects were exposed to higher partial pressures of oxygen, which seem to have more firmly anchored their baseline $\mathrm{SpO}_{2}$ readings in the $99-100 \%$ range. This effect indicates that any pulse oximeter device that is developed and assessed preliminarily during testing at surface pressures will actually perform better at depth, with fewer false positives.

An underwater pulse oximeter-based alarm device would provide a useful biomedical intervention that is fully independent from and therefore redundant to the rebreather electronics. This project started as some equations scribbled on the back of a piece of scrap paper after the accidental death of National Oceanographic and Atmospheric Association (NOAA) employee Dewey Smith in 2009. His rebreather was switched off without his knowledge by the action of nearby underwater tools, causing a fatal accident that emphasized that no level of skill can fully eliminate the risks of rebreather diving. With the permission of his family, we request that anyone using this concept to develop further devices for the monitoring of rebreather divers refer to them as "Dewey Monitors" in his honor.

\section{ACKNOWLEDGMENTS}

We would like to thank our funding sponsors, the Naval Sea Support Command (NAVSEA) for providing the funds to conduct this work (Award No. N0002418CA317). This project and concept has been especially supported by Capt David Regis (USN), Capt Ted Waters (USN), and Capt (Ret) David Southerland (USN), without whose support, understanding, and insight the work would have been far more difficult. We would also like to acknowledge the engineering work of Brian Wentworth, Bryan Le, and Tye Langston of Code 15 Underwater Systems Development and Acquisition of Naval Surface Warfare Center Panama City Division (NSWC PCD). Fabio Di Pumpo's contributions to this work were sufficient and valuable enough to warrant co-authorship, but we would also like to thank the Italian Navy and Divers and Raiders Group Command "Teseo Tesei" COMSUBIN for loaning us their sensors and his expertise and time. And as always, the rest of the staff and faculty of the Duke Center for Hyperbaric
Medicine \& Environmental Physiology have served to support the researchers on this paper as our teammates in all scientific endeavors.

\section{CONFLICT OF INTEREST}

No benefits in any form have been or will be received from a commercial party related directly or indirectly to the subject of this manuscript.

\section{REFERENCES}

${ }^{1}$ Di Pumpo, F., G. Ruffino, and P. Malacarne. Pulse oximeter to detect peripheral oxygen saturation in underwater rebreather ECCR diver: a preliminary study. Undersea Hyperbaric Med. 46(1):1-6, 2019.

${ }^{2}$ Fock, A. W. Analysis of recreational closed-circuit rebreather deaths 1998-2010. Div. Hyperb. Med. 43(2):78-85, 2013.

${ }^{3}$ Hans Rudolph, Inc. Data Sheet for Manual Directional Control Valves Three-Way T-Shape Stopcock-Type Series 2130, 2120, 2110, 2100 (Rev H). https://www.rudolphkc.c om/img/uploads/pdf/691004\%201013\%20H.pdf. Accessed 15 Oct 2013.

${ }^{4}$ Island, R., and E. Fraley. Analysis of USAF hypoxia incidents January 1976 through March 1990. In: 7th International Symposium on Aviation Physiology, Columbus, OH, USA, pp. 664-668, 1993.

${ }^{5}$ Komarov, V. Open Revolution Accident Review of Life Support Systems: Rebreather Fatal Accident Database. w ww.deeplife.co.uk: Deep Life Ltd.

${ }^{6}$ Lance, R. M., M. J. Natoli, S. A. S. Dunworth, J. J. Freiberger, and R. E. Moon. The Dewey Monitor: Pulse oximetry can independently detect hypoxia in a rebreather diver. Undersea Hyperbaric Med. 44(6):569-580, 2017.

${ }^{7}$ MacLeod, D. B., L. I. Cortinez, J. C. Keifer, D. Cameron, D. R. Wright, W. D. White, et al. The desaturation response time of finger pulse oximeters during mild hypothermia. Anaesthesia. 60:65-71, 2005.

${ }^{8}$ Molecular Products Safety Data Sheet (SDS), Sofnolime (Ref 23, v21). https://www.molecularproducts.com/wp-co ntent/uploads/2021/05/Ref-23-Sofnolime-SDS-English-v21 .pdf. Accessed 1 Jun 2020.

${ }^{9}$ Mulder, E., A. Sieber, and E. Schagatay. Using underwater pulse oximetry in freediving to extreme depths to study risk of hypoxic blackout and diving response phases. Front Physiol. 12:1-12, 2021.

${ }^{10}$ Stewart, I. B., A. C. Bulmer, J. E. Sharman, and L. Ridgway. Arterial oxygen desaturation kinetics during apnea. Med. Sci. Sports Exerc. 37(11):1871-1876, 2005.

${ }^{11}$ USN. U.S. Navy diving manual, rev. 6. 2011.

Publisher's Note Springer Nature remains neutral with regard to jurisdictional claims in published maps and institutional affiliations. 